\title{
Costo directo del retraso de una cirugía de urgencia de columna toracolumbar clasificadas (AO) B o C
}

\author{
Direct cost of the delay of an urgency surgery of the thoracolumbar spine classified $(A O) B$ or $C$ \\ José de Jesús González-Mercado, ${ }^{*}$ Bernardo Ramírez-Serratos, ${ }^{*}$ \\ Arelhi Catalina González-Cisneros, ${ }^{\ddagger}$ José María Jiménez-Ávila* \\ *Unidad Médica de Alta Especialidad. Centro Médico Nacional de Occidente. Hospital de Especialidades. Servicio de Columna. Guadalajara, Jalisco, México; \\ ‡Escuela de Medicina. Instituto Tecnológico y de Estudios Superiores de Monterrey, Campus Guadalajara.
}

\begin{abstract}
Resumen
Introducción: El alto costo de una cirugía de columna, su hospitalización y pronóstico generan una amplia importancia económica para el sistema de salud, por lo cual es primordial el conocimiento de su manejo como enfermedad prioritaria. Objetivo: Determinar el costo del retraso de la cirugía de columna de urgencia. Material y métodos: Se calculó el costo del retraso de una cirugía de columna de urgencia mediante un aproximado de días de hospitalización y complicaciones. La muestra consistió en 115 pacientes con fractura de columna tipo B o C de la clasificación de la AO entre los años 2014-2019. Se evaluó edad, comorbilidades, mecanismo de lesión, clasificación, estado neurológico, complicaciones, retraso de cirugía y costos de hospitalización. Resultados: De los 115 pacientes 75 presentaron lesión de columna torácica (75.3\%) y 40 lesión de lumbares (34.7\%). La hospitalización promedio fue de 11.9 días. Cuarenta por ciento tuvo retraso de cirugía y las enfermedades infecciosas fueron las principales causas de complicaciones. Los pacientes que tuvieron larga estancia presentaron $30 \%$ del total del aumento de los costos. Conclusión: El aumento en complicaciones y en costos totales de pacientes con retraso de cirugía de urgencia es claro, por lo que se debe optimizar la atención para los pacientes de urgencias.
\end{abstract}

Palabras clave: Cirugía de columna de urgencia, fractura $\mathrm{AO}$ tipo $\mathrm{B}$, fractura $\mathrm{AO}$ tipo $\mathrm{C}$, costos de retraso de cirugía.

\begin{abstract}
Introduction: The high cost of spinal surgery, its hospitalization and prognosis, generate a wide economic importance for the health system, being a priority the knowledge of its management as a priority disease. Objective: To determine the cost of the delay of emergency spine surgery. Material and methods: The cost of delaying emergency spinal surgery was calculated through an approximate number of days of hospitalization and complications. Sample: 115 patients with type B or C spinal fracture of the AO classification between the years 2014-2019. The following were evaluated: age, comorbidities, injury mechanism, classification, neurological status, complications, and delay in surgery and hospitalization costs. Results: Of the 115 patients: 75 had a thoracic spine injury (75.3\%) and 40 a lumbar injury (34.7\%). Average hospitalization days: 11.9 days. 40\% delayed surgery, infectious diseases being the main causes of complications. Long-stay patients accounted for $30 \%$ of the total cost increase. Conclusion: The increase in complications and in total costs of patients with delayed emergency surgery is clear, so care must be optimized for emergency patients.
\end{abstract}

Keywords: Emergency spine surgery, $A O$ type $B$ fracture, $A O$ type $C$ fracture, surgery delay costs.

\section{Introducción}

Los gastos de salud pública son de gran importancia, se ha demostrado que la disminución de costos puede ser parte de la mejoría del pronóstico del paciente. El redescribir los costos de

Correspondencia:

José de Jesús González-Mercado

E-mail: dr.jesusgonzalez26@gmail.com

Recibido: 14-02-2021. Aceptado: 15-02-2021. una enfermedad o su tratamiento adecuado en tiempo y forma puede ahorrar una gran cantidad de gastos no necesarios; sin embargo, la clave es identificar cuáles son los gastos innecesarios de una enfermedad, entendiendo como innecesarios aquellos que no modifican el pronóstico de forma negativa en el tratamiento.

Citar como: González-Mercado JJ, Ramírez-Serratos B, GonzálezCisneros AC, Jiménez-Ávila JM. Costo directo del retraso de una cirugía de urgencia de columna toracolumbar clasificadas (AO) B o C. Orthotips. 2021; 17 (1): 11-17. https://dx.doi.org/10.35366/99162 
En el ámbito internacional la forma más común de comparar los gastos es determinado por el porcentaje del producto interno bruto (PIB) que representa el gasto nacional en salud (GNS). Entre los mecanismos más comunes de financiamiento del sector sanitario figuran los fondos públicos, los seguros de salud, el cobro a los usuarios, la seguridad social y la cooperación internacional. ${ }^{1}$

En algunos países la evaluación económica se utiliza de forma explícita y sistemática para establecer el precio y el financiamiento de medicamentos y tecnologías sanitarias para emitir recomendaciones acerca del uso de las mismas. ${ }^{2}$ En Estados Unidos las lesiones se ubican en el sexto lugar en mayor gasto de salud pública, siendo las principales lesiones las fracturas de columna toracolumbar, con o sin lesión medular. ${ }^{3}$

De igual manera, se evalúa la asociación entre el retraso de una cirugía de urgencia con la mortalidad y el gasto de salud pública. Como conclusiones se demuestra que la morbilidad aumenta cuando se retrasa una cirugía de urgencia y que los costos totales aumentan de manera directa en más de $15 \% .{ }^{4}$ Es evidente que los costos son mayores al retrasar una cirugía de urgencia; sin embargo, en fracturas de columna toracolumbar también existen estudios que evalúan el costo de cirugía temprana versus tardía. Las cirugías tempranas pueden ser mucho más económicas a corto plazo. ${ }^{5}$

En casos de politraumatizados, las lesiones de columna se presentan hasta en $31 \%$ de pacientes que presentan múltiples fracturas o alta energía. ${ }^{6}$ La lesión de la médula espinal se considera el segundo evento traumático más grave después de la lesión cerebral traumática en términos de morbilidad y discapacidad. ${ }^{7}$ Los mecanismos de trauma de la lesión de la médula espinal fueron caídas en $44 \%$, accidentes de tránsito en $36.5 \%$, accidentes de buceo en $8.9 \%$ y otros en $10.7 \% .{ }^{8}$ Por lo general, las fracturas toracolumbares se deben a traumatismos de alto impacto, en su mayoría accidentes automovilísticos o caídas, seguidas en frecuencia por heridas penetrantes, ya sea por arma de fuego o traumatismos directos por violencia delictiva o deportes de contacto. ${ }^{9}$

En 2003 en México se publicó un estudio en el que se reporta 1.79 casos por cada 100,000 habitantes con $79 \%$ de masculinos y $21 \%$ de femeninos con un promedio de edad de 36 años, siendo la primera causa las caídas y en segundo lugar los accidentes automovilísticos, además, el primer tipo de lesión fueron las lumbares, segundo las cervicales y tercero las torácicas. ${ }^{10}$
Por otra parte, una revisión en la que se habla de un gasto en politraumatizados demuestra que el gasto por paciente con fractura de columna se vuelve relevante para la evaluación económica, ya que este es un procedimiento de alto costo. ${ }^{11}$

Asimismo, existen múltiples artículos que evalúan los costos de las fracturas de columna cervical y comparan el tratamiento quirúrgico contra el no quirúrgico. Se calculó el costo directo aproximado que representó para la institución. El costo total (en pesos) de los pacientes que sobrevivieron fue de $\$ 2,536,115.59$, lo cual corresponde a $29.4 \%$ del costo total y un costo de $\$ 6,086,677.41(70.6 \%)$ en pacientes que fallecieron. ${ }^{12}$ De igual modo, se comparó la cirugía de manera temprana contra la tardía y sus costes. Los resultados sugieren que la descompresión temprana fue más rentable que la tardía. ${ }^{13}$ Además, se demuestra que la intervención quirúrgica temprana disminuye los costes de manera directa e incluso las complicaciones. ${ }^{14}$

Con respecto al costo de los pacientes que no fueron tratados quirúrgicamente, el principal gasto es el tiempo de hospitalización, ${ }^{15}$ en vista de la rentabilidad es preferible la terapia quirúrgica de las fracturas traumáticas de la columna toracolumbar. ${ }^{16}$ La cantidad de costo directo de un trauma de columna es bastante amplia. ${ }^{17}$

Los costos totales de la lesión cerebral traumática y de la lesión de médula espinal se estimaron en \$8.6 mil millones y $\$ 2.0$ mil millones, respectivamente, siendo el costo financiero de la tetraplejia más altos que todas las condiciones de comparación. ${ }^{18}$ En la estructura de costes, la partida de mayor peso es la referida al coste hospitalario, que supone casi $83 \%$ del coste total estimado. ${ }^{19}$

Son más comunes las lesiones en hombres (4:1) que en mujeres. La estancia hospitalaria tuvo una mediana de 14 días y el costo promedio de hospitalización fue de 2,874.80 USD, con un aumento de gasto total en pacientes que presentaron infección. ${ }^{20}$

Las complicaciones intrahospitalarias de las lesiones medulares también son relevantes, poseen gran impacto en los costos por aumento de días de hospitalización, medicamentos 0 atención de otros especialistas. ${ }^{21}$ De acuerdo con un estudio de incidencia de infecciones nosocomiales en la unidad de lesiones medulares, los resultados obtenidos son relevantes para las complicaciones y los costos de la enfermedad. ${ }^{22}$

El aumento de gastos económicos en el retraso de la cirugía parecer ser inminente; sin embargo, deberá ser estudiado en cada población para poder 
crear alternativas que disminuyan el mismo. Al tomar esta premisa como hipótesis el grupo de estudio busca demostrar que puede ser disminuida la cantidad de gastos totales con la atención prioritaria de esta enfermedad; buscando evitar cualquier factor de retraso de ésta.

\section{Material y métodos}

Se trata de un estudio de serie de casos en el que se incluyeron pacientes que sufrieron fractura toracolumbar tipo B o C de acuerdo con la clasificación de la Fundación AO para fracturas toracolumbares entre enero de 2014 a enero de 2020 . Se excluyeron a pacientes que no fueron operados debido a su fallecimiento antes de la cirugía (esto será visto como complicación en pacientes postquirúrgicos). Los criterios de eliminación fueron aquellos pacientes que no contaron con los datos de variables terminados. Se tuvo un cálculo de muestra de 115 pacientes, sin necesidad de reposición de pérdidas; se evaluó edad, sexo, comorbilidades, tipo de traumatismo, fecha y hora de traumatismo, fecha y hora de arribo al hospital, nivel de fractura, clasificación de fractura, nivel vertebral de fractura, grado de lesión medular, fecha y hora de cirugía, niveles instrumentados quirúrgicamente, complicaciones, días de hospitalización y costos de hospitalización. Se utilizó la clasificación para fracturas toracolumbares de la Fundación $\mathrm{AO}^{23}$ (Tabla 1) y para valorar la lesión medular del paciente fue la escala ASIA (American Spinal Injury Association) ${ }^{24}$ (Tabla 2); además, se realizó un análisis descriptivo en el que se evaluaron frecuencias absolutas y relativas para variables cualitativas, y promedio y desviación estándar para las variables cuantitativas.

Tabla 1: Clasificación AO para fracturas toracolumbares.

\begin{tabular}{|c|c|c|c|c|}
\hline \multicolumn{5}{|c|}{ Clasificación para fracturas toracolumbares } \\
\hline \multicolumn{2}{|l|}{ A } & \multicolumn{2}{|l|}{ B } & $\mathrm{C}$ \\
\hline \multicolumn{2}{|c|}{$\begin{array}{l}\text { Describe daño del cuerpo vertebral sin } \\
\text { dañar la banda de tensión }\end{array}$} & \multicolumn{2}{|c|}{$\begin{array}{l}\text { Describe la falla de complejo ligamentario posterior } \\
\text { o ligamento longitudinal anterior (esta lesión puede } \\
\text { estar asociada con cualquier fractura de tipo A) }\end{array}$} & $\begin{array}{l}\text { Describe desplazamiento o luxación (esta lesión } \\
\text { puede estar asociada con cualquier fractura de } \\
\text { tipo A) }\end{array}$ \\
\hline A0 & $\begin{array}{l}\text { Se usa para describir frac- } \\
\text { turas insignificantes que no } \\
\text { afectan la estabilidad espinal } \\
\text { de manera significativa, como } \\
\text { una fractura aislada del } \\
\text { proceso espinoso, el proceso } \\
\text { transversal o la lámina }\end{array}$ & B1 & $\begin{array}{l}\text { Es una lesión de la banda de ten- } \\
\text { sión posterior ósea monosegmental } \\
\text { Hay una falla ósea completa de } \\
\text { la banda de tensión posterior en } \\
\text { un nivel que se extiende hacia el } \\
\text { cuerpo vertebral }\end{array}$ & $\begin{array}{l}\text { Implican una interrupción o separación de } \\
\text { los componentes anterior y posterior de los } \\
\text { segmentos vertebrales. La interrupción del } \\
\text { componente anterior puede ser a través del } \\
\text { disco o del cuerpo vertebral. La interrupción de } \\
\text { los elementos posteriores puede ser a través del } \\
\text { proceso espinoso, lámina, para interarticulares o } \\
\text { complejo ligamentoso posterior }\end{array}$ \\
\hline A1 & $\begin{array}{l}\text { Son fracturas del cuerpo } \\
\text { vertebral con una o ambas } \\
\text { placas terminales involucra- } \\
\text { das, pero las fracturas no se } \\
\text { conectan entre sí }\end{array}$ & B2 & $\begin{array}{l}\text { El subtipo B2 es una ruptura de la } \\
\text { banda de tensión posterior con falla } \\
\text { a través de los tejidos blandos y los } \\
\text { elementos óseos }\end{array}$ & \\
\hline A2 & $\begin{array}{l}\text { Son fracturas del cuerpo } \\
\text { vertebral en las que la frac- } \\
\text { tura involucra ambas placas } \\
\text { terminales }\end{array}$ & B3 & $\begin{array}{l}\text { Se caracteriza por una lesión } \\
\text { por tensión del disco o el cuerpo } \\
\text { vertebral o ambos, lo que resulta } \\
\text { en una posición hiperextendida de } \\
\text { la columna vertebral. El ligamento } \\
\text { longitudinal anterior está roto }\end{array}$ & \\
\hline A3 & $\begin{array}{l}\text { Son fracturas de una sola pla- } \\
\text { ca terminal. Puede haber una } \\
\text { fractura vertical de la lámina. } \\
\text { La banda de tensión posterior } \\
\text { se mantiene }\end{array}$ & & & Y \\
\hline A4 & $\begin{array}{l}\text { Son fracturas de ambas } \\
\text { placas terminales. Una frac- } \\
\text { tura vertical de la lámina no } \\
\text { implica ruptura de la banda de } \\
\text { tensión posterior }\end{array}$ & & & \\
\hline
\end{tabular}


Tabla 2: Escala American Spinal Injury Association (ASIA).

\begin{tabular}{l}
\hline Clasificación ASIA \\
\hline Establece las definiciones básicas de los términos usados en la valoración de la lesión medular y establece una clasificación de acuerdo con cinco \\
grados determinados por la ausencia o preservación de la función motora y sensitiva, indicando la severidad de dicha lesión y su posible pronósti- \\
co basado en la evaluación por dermatomas (sensibilidad) y miotomas (motricidad) \\
\hline A $\quad$ Ausencia de función motora y sensitiva que se extiende hasta los segmentos sacros S4-S5 \\
B $\quad \begin{array}{l}\text { Preservación de la función sensitiva por debajo del nivel neurológico de la lesión que se extiende hasta los segmentos sacros S4-S5 y con } \\
\text { ausencia de función motora }\end{array}$ \\
C $\quad \begin{array}{l}\text { Preservación de la función motora por debajo del nivel neurológico y más de la mitad de los músculos llave, pero debajo del nivel neuroló- } \\
\text { gico tienen un balance muscular menor de tres }\end{array}$ \\
Preservación de la función motora por debajo del nivel neurológico y más de la mitad de los músculos llave por debajo del nivel neurológi- \\
E co tienen un balance muscular de tres o más
\end{tabular}

\section{Resultados}

Se analizaron a 115 pacientes con fractura tipo B o $\mathrm{C}$ de la clasificación $\mathrm{AO}$ de fracturas toracolumbares. En distribución de sexo 91 pacientes fueron masculinos $(79.2 \%)$ y 24 femeninos (20.8\%). En cuanto a la edad, existe un promedio de 37 años con una desviación estándar de 14 años (Figura 1).

Con respecto a la sección de columna lesionada, 75 de los pacientes tienen lesión de columna torácica (75.3\%) y 40 presentaron lesión de lumbares (34.7\%). El diagnóstico más común en la columna torácica fue la lesión tipo $\mathrm{C}$ con 43 pacientes (57.3\%), seguido de la lesión tipo B1 con 17 pacientes (22.6\%), en tercer lugar la lesión B2 con 14 pacientes (18.6\%) y sólo un paciente con lesión B3 (1.3\%). En las lesiones de la columna lumbar el tipo de lesión más común fue el B2 con 17 casos (42.5\%), en segundo lugar el tipo C con 11 pacientes $(27.5 \%)$, después la lesión B1 con 10 casos (25\%) y por último el tipo B3 con dos pacientes $(5 \%)$ (Figura 2).

La distribución etaria fue analizada con grupos de cinco años: de 16 a 20 años se presentan 14 pacientes (12.1\%); de 21 a 25 años, 18 casos (15.6\%), siendo el grupo mayor; de 26 a 30 años existen 17 pacientes (14.7\%); de 31 a 35 años, seis casos (5.2\%); de 36 a 40 años se cuenta con 13 pacientes (11.3\%); de 41 a 45 años, con 10 casos (8.6\%); de 46 a 50 años posee siete pacientes (6\%); de 51 a 55 años, 14 casos (12.1\%); de 56 a 60 años existen ocho pacientes (6.9\%); de 61 a 65 años, cinco casos (4.3\%); y tres pacientes son mayores a 65 años (2.6\%): un paciente de 68 y dos de 72 años. Se encuentra una media de edad de 37.7 años y una desviación estándar de 14.8 años.

En cuanto a la distribución de los pacientes en relación con la fecha de ocurrencia, se presentó sólo un paciente en 2014 (0.8\%); en 2015 fueron 11 pacientes (9.5\%); 19 pacientes en 2016 (13.9\%); 26 casos en 2017 (22.6\%); el año con mayor presentación fue 2018 con 39 pacientes (33.9\%) y 2019 presentó 19 casos (16.5\%).

Las comorbilidades que mayormente presentaron fueron: «otras» con 40 sujetos (34.7\%), 38 pacientes eran fumadores (33\%), 16 tenían algún tipo de obesidad (13.9\%), la diabetes mellitus tipo 2 se presentó en 10 pacientes $(8.6 \%)$, siete presentaban hipertensión $(6 \%)$, tres pacientes presentaban enfermedades

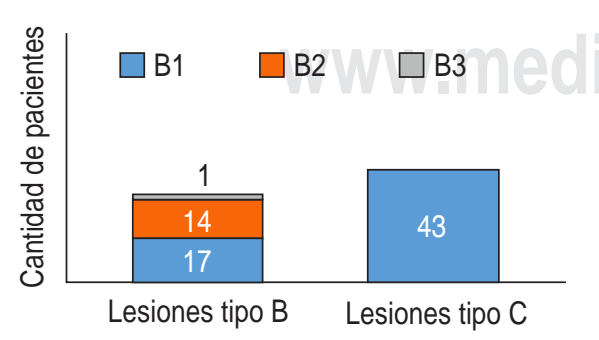

Figura 1: Lesión de columna torácica.

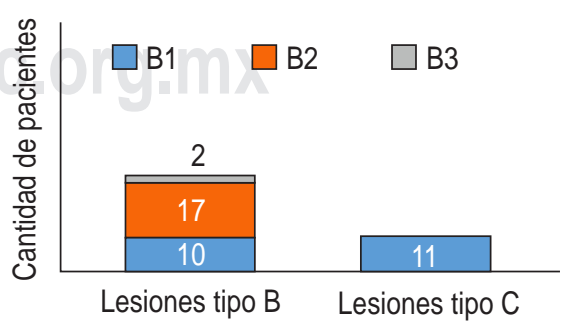

Figura 2: Lesión de columna lumbar. 
pulmonares $(2.6 \%)$ y sólo un paciente presentó problemas cardiacos $(0.86 \%)$.

El mecanismo de lesión más común fueron las caídas de altura con 55 pacientes (47.8\%), 25 sufrieron accidentes de motocicleta (21.7\%), el accidente en automóvil fue el causante de lesión en 23 pacientes (20\%) y 12 presentaron otra causa de lesión (10.4\%).

La vértebra más fracturada fue la $L 1$ con 22 pacientes (19.1\%), por ende, la más común en columna lumbar y en la columna torácica fue T12 con 18 pacientes (15.6\%) (Figura 3).

El déficit neurológico fue evaluado con la escala ASIA, la clasificación mayormente presentada fue la $A$ con 56 pacientes (48.6\%), E con 37 (32.1\%), C con 10 $(8.6 \%)$, D con siete $(6 \%)$ y B con cinco casos $(4.3 \%)$.

La hospitalización promedio fue de 11.9 días por paciente en general con una desviación estándar de 10.3 días. En pacientes con retraso de cirugía mayor a 10 días (los cuales fueron 57 [49.67\%]) sus días de hospitalización promedio fue de 21.7; mientras que a los pacientes con un retraso de cirugía menor a nueve días (quienes fueron 58 [50.43\%]) tuvieron un promedio de 4.9 días.

Cuarenta pacientes (34.7\%) presentaron algún tipo de complicación: cinco pacientes tuvieron neumonía $(4.3 \%), 16$ pacientes infección urinaria $(13.9 \%)$, cuatro pacientes infección de herida quirúrgica (3.4\%), sólo un paciente tuvo que ser reintervenido, tres pacientes sufrieron de embolismo pulmonar (2.6\%), dos pacientes presentaron dehiscencia de herida (1.7\%), tres pacientes fallecieron (2.6\%) y seis pacientes presentaron otro tipo de complicaciones (5.2\%).

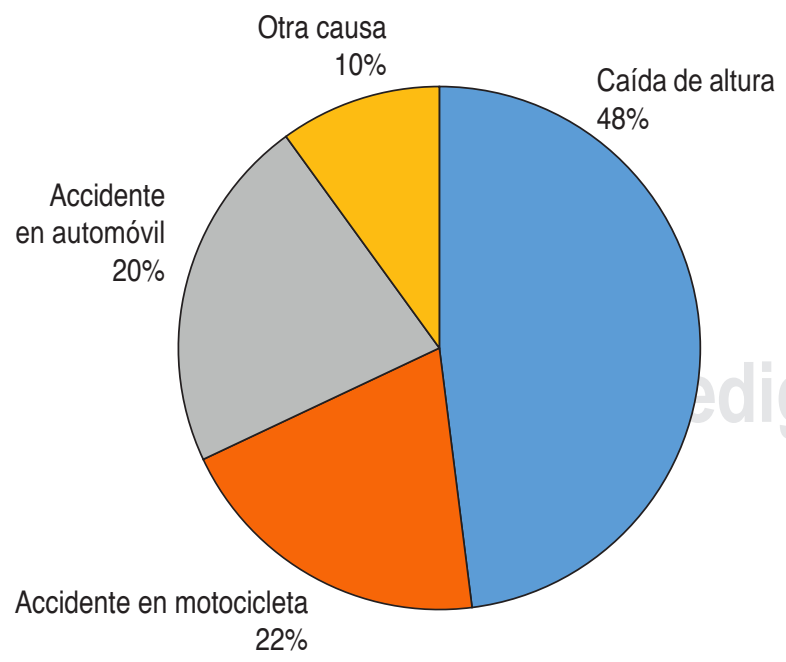

Figura 3: Mecanismo de lesión.

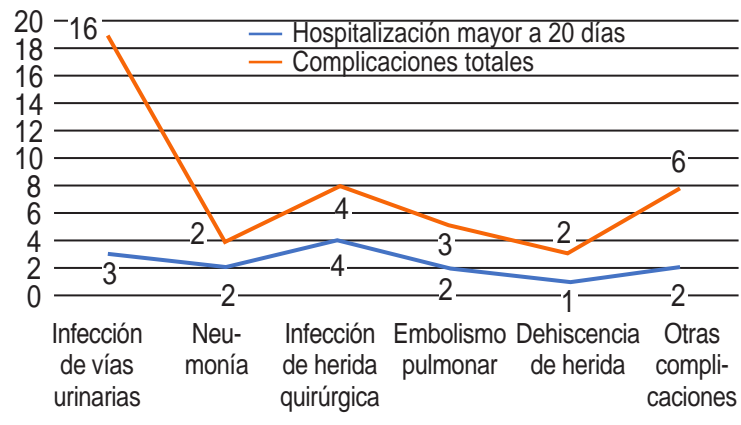

Figura 4: Complicaciones.

Un grupo de 21 pacientes (24.1\%) se mantuvieron hospitalizados por más de 20 días, de los cuales 12 (57.1\%) prolongaron su estancia por complicaciones: tres de ellos presentaron infección de vías urinarias (25\%), dos tuvieron neumonía (16.6\%), dos infección de herida quirúrgica (16.6\%), dos embolismo pulmonar $(16.6 \%)$, un paciente presentó dehiscencia de herida $(8.3 \%)$ y dos pacientes más presentaron otro tipo de complicaciones (16.6\%) (Figura 4).

Los pacientes que tienen un retraso de cirugía mayor a 10 días son 45 (39\%), 18 de estos tienen una hospitalización mayor a 20 días $(40 \%)$ y nueve de esos $18(50 \%)$ se relaciona su larga estancia por complicaciones, para el resto de los sujetos no será evaluada la causa de retraso, sino sólo el coste económico que éste generó.

El costo (en pesos mexicanos) por días de hospitalización en promedio fue de $\$ 11,322,266.90$ en los cinco años; 2014 presentó un gasto de $\$ 75,886.30$; 2015 tuvo un total de $\$ 881,087.90$; 2016 mostró un gasto de $\$ 2,183,673.80 ; 2017$ presentó un gasto de $\$ 2,245,006.40 ; 2018$ mostró un gasto de $\$ 3,867,345.30$ y 2019 con $\$ 1,974,305.20$, presentando un promedio de gasto anual de $\$ 1,887,044.48$ con una desviación estándar de $\$ 1,356,293.95$ (Figura 5).

El costo total por días de hospitalización no promedio fue de $\$ 11,349,649$ en los cinco años; 2014 presentó un gasto de $\$ 133,917 ; 2015$ mostró un total de $\$ 437,515 ; 2016$ tuvo un gasto de $\$ 1,468,016$; 2017 presentó un gasto de $\$ 2,416,248 ; 2018$ mostró un gasto de $\$ 3,885,740$ y 2019 tuvo un gasto de $\$ 3,008,213$, presentando un promedio de gasto anual de $\$ 1,891,608.16$ con una desviación estándar de $\$ 1,475,256.62$.

El costo de los pacientes que han sufrido un retraso en su cirugía mayor a 10 días (contado por días no promedio) fue de $\$ 6,363,310$ en los cinco años, 
lo cual representa $56 \%$ del costo total no promedio; 2014 presentó un gasto de $\$ 215,392 ; 2015$ tuvo un total de $\$ 215,392 ; 2016$ mostró un gasto de $\$ 647,086$; 2017 presentó un gasto de $\$ 1,603,576 ; 2018$ tuvo un gasto de $\$ 1,571,760$ y 2019 mostró un gasto de $\$ 2,191,579$, presentando un promedio de gasto anual de $\$ 1,060,551.66$ con una desviación estándar de $\$ 846,088,97$ (Figura 6).

\section{Discusión}

El retraso en la cirugía de urgencia de columna es un factor relevante de costos, ya que al evaluar el total no promedio de costos de hospitalización de los pacientes evaluados y el costo de quienes permanecen hospitalizados por mayor tiempo (más de 10 días) es más de la mitad de todos los costos, lo cual demuestra que el retraso es contraproducente económicamente.

Múltiples artículos exponen que la suspensión de cirugías tiene un aumento de costos de manera directa, pero en nuestro estudio el aumento total es de casi $30 \%$ en comparación con los artículos mencionados anteriormente, los cuales hablan de un aumento de $15 \%$ en costos, lo que demuestra que el aumento es mucho mayor sin contar las complicaciones. Esto revela que el retraso de la cirugía de urgencia debería ser una prioridad de salud pública dentro de todos los hospitales.

El aumento de los costos es amplio, por lo que se deberían buscar opciones que agilicen las cirugías de

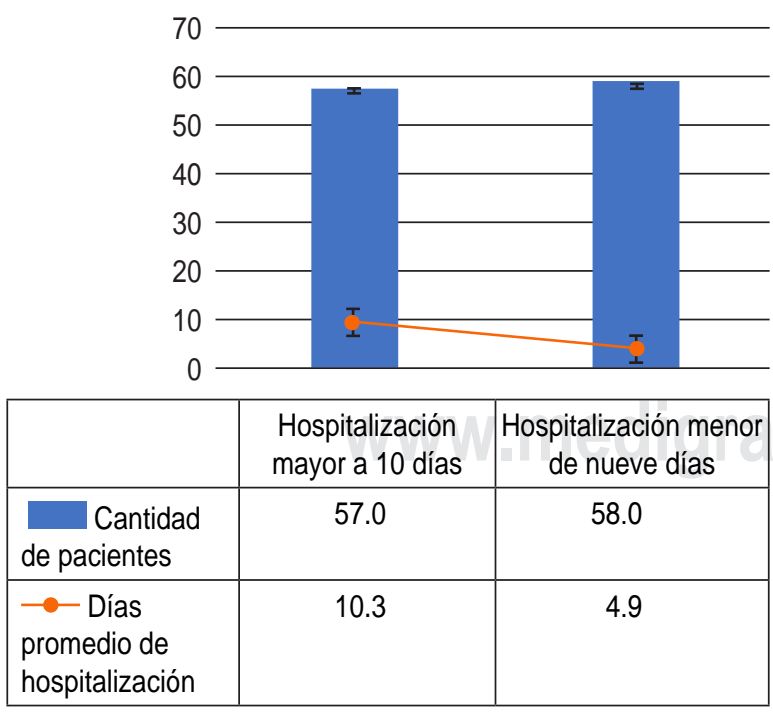

Figura 5: Hospitalización.

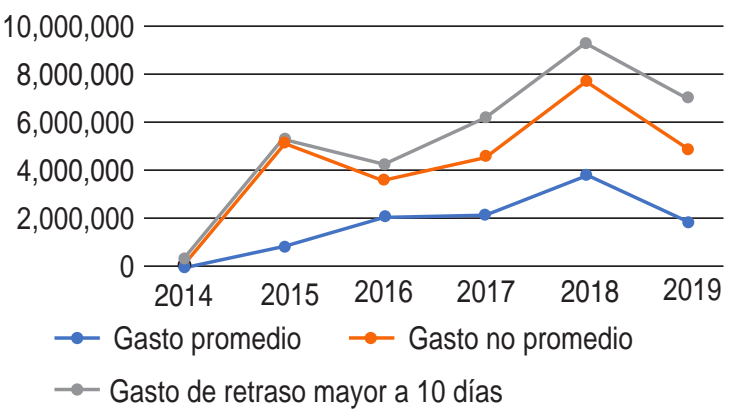

Figura 6: Gastos anuales.

urgencias para disminuir la cantidad de retrasos en cuanto a aspectos administrativos.

El incremento de complicaciones es notorio dentro de nuestro artículo, ya que $30 \%$ de todos los pacientes del estudio presentan alguna complicación (las principales son las de origen infeccioso), las cuales aumentan los costes por estancia hospitalaria y medicamentos. De igual manera, la mitad de los pacientes que retrasan su cirugía con una larga estancia mayor a 20 días presentan complicaciones, lo que da una relación clara entre el retraso de cirugía con el aumento de complicaciones.

La cantidad de pacientes permite que sea una muestra de tamaño relevante; no obstante, el aumento de costos es aproximado a cantidades prefijas por el Instituto Mexicano del Seguro Social.

\section{Conclusión}

El aumento en costos, complicaciones y empeoramiento del pronóstico en los pacientes en los que se retrasa una cirugía de urgencia debe tener atención por parte de cualquier hospital para optimizar el uso de recursos y mejorar el pronóstico de sus pacientes, ya que se generan años potencialmente perdidos debido a que entre más pronto se opere a un paciente, el costo será menor y el pronóstico del paciente también.

Asimismo, es de suma importancia sumar esfuerzos en la optimización de recursos, así como en establecer protocolos y directrices de manejo para la pronta realización de los procedimientos quirúrgicos en la patología de columna traumática.

\section{Referencias}

1. Collazo HM, Cárdenas RJ, González LR, Abreu RM, Gálvez GA, Casulo JC. La economía de la salud: ¿debe ser de interés 
para el campo sanitario? Rev Panam Salud Pública. 2002; 12: 359-365.

2. Dilla T, González de Dios J, Sacristán JA. Evaluación económica en medicina (I): fundamentos y metodología. Evid Pediatr. 2009; 5: 71.

3. Dieleman JL, Baral R, Birger M, Bui AL, Bulchis A, Chapin A, et al. US spending on personal health care and public health, 1996-2013. JAMA. 2016; 316 (24): 2627-2646.

4. Mclsaac DI, Abdulla K, Yang $H$, Sundaresan S, Doering $P$, Vaswani SG, et al. Association of delay of urgent or emergency surgery with mortality and use of health care resources: a propensity score-matched observational cohort study. CMAJ. 2017; 189 (27): E905-E912.

5. Boakye M, Arrigo RT, Hayden GM, Zygourakis CC, Lad S. Retrospective, propensity score-matched cohort study examining timing of fracture fixation for traumatic thoracolumbar fractures. J Neurotrauma. 2012; 29 (12): 2220-2225.

6. Montmany S, Navarro S, Rebasa P, Hermoso J, Hidalgo JM, Cánovas G. Estudio prospectivo de la incidencia de las lesiones inadvertidas en el paciente politraumatizado. Cirugía Española. 2008; 84 (1): 32-36.

7. Van den Berg M, Castellote JM, Mahillo-Fernández I, de Pedro J. Incidencia de lesión traumática de la médula espinal en Aragón, España. J Neurotrauma. 2011; 28: 469-477.

8. Bárbara-Bataller $E$, Méndez-Suárez J, Alemán-Sánchez $C$, Sánchez-Enríquez J, Sosa-Henríquez M. Change in the profile of traumatic spinal cord injury over 15 years in Spain. Scan $J$ Trauma Resusc Emerg Med. 2018; 26 (1): 27.

9. Vinas FC. Lumbar spine fractures and dislocations. Medicine Specialties. 2009.

10. Cisneros-Dreinhofer F, Hurtado-Padilla A. Incidencia de las lesiones vertebrales traumáticas en el Servicio de Cirugía de Columna de un hospital de concentración del sistema de seguridad social. Acta Ortop Mex. 2003; 17 (6): 292-297.

11. Aras EL, Bunger C, Hansen ES, Slogaard R. Cost-effectiveness of surgical versus conservative treatment for thoracolumbar burst fractures. Spine. 2016; 41 (4): 337-343.

12. Jiménez-Ávila JM, Álvarez-Garnier JC, Bitar-Alatorre WE. Costo directo de la lesión medular completa de la columna cervical. Acta Ortop Mex. 2012; 26 (1): 10-14.

13. Ferrara DM. Estudio de variables clínicas, resultados en salud y análisis coste - utilidad en pacientes intervenidos de una recidiva de hernia de disco lumbar. [Tesis Doctoral]. Universidad de Castilla de la mancha. 2017.
14. Medress Z, Arrigo RT, Hayden GM, Zygourakis CC, Boakye M. Cervical fracture stabilization within 72 hours of injury is associated with decreased hospitalization costs with comparable perioperative outcomes in a propensity scorematched cohort. Cureus. 2015; 7 (1): e244.

15. Van der Roer N, De Bruyne MC, Bakker FC, Van Tulder MW, Boers M. Direct medical costs of traumatic thoracolumbar spine fractures. Acta Orthop. 2005; 76 (5): 662-666.

16. Siebenga J, Leferink VJ, Segers MJ, Elzinga MJ, Bakker FC, Haarman $\mathrm{HJ}$, et al. Treatment of traumatic thoracolumbar spine fractures: a multicenter prospective randomized study of operative versus nonsurgical treatment. Spine. 2006; 31 (25): 2881-2890.

17. Jiménez-Ávila JM, Calderón-Granados A, Bitar-Alatorre WE. Costo directo de las lesiones en la columna. Cir Cir. 2012; 80 (5): 435-441.

18. Collie A, Keating C, Pezzullo L, Gabbe B, Cooper J, Brown D, et al. Brain and spinal cord injury in Australia-economic cost and burden of disease. Injury Prevention. 2010; 16 (Suppl 1): A25-A26.

19. Rodríguez GA. Estimación de los costes sociales asociados a los lesionados medulares [Licenciatura]. Universidad de Coruña; 2016.

20. Ludwig NT, Willimann RL, Martins RM, Pelegrini AL, Finger G, Frizon GG, et al. Thoracolumbar spinal arthrodesisepidemiology and costs. Coluna/Columna. 2017; 16 (1): 52-55.

21. Del Busto JEB, González EH, Reinaldo MP, Suárez FA, Pérez FZ. Caracterización clínica de pacientes con lesión medular traumática, Rev Mex Neuroci. 2002; 3 (3): 135-142.

22. Girona BL, Castelló VT, Gol VV. Incidencia y coste de las infecciones nosocomiales en una unidad de lesionados medulares. Farmacia Hospitalaria. 2001; 25 (4): 215-223.

23. Tejeda BM. Clasificación de fracturas toracolumbares. Orthotips. 2010; 6 (2): 114-121.

24. Torre GD, Pérez MJA, Góngora LJ, Huerta OVM. Evaluación de pacientes con traumatismo raquimedular clínico y radiológico. Trauma. 2001; 4 (1): 22-28.

\section{Conflicto de intereses}

El autor y coautores no tienen ningún conflicto de intereses en relación a esta investigación. 